\title{
Pregnancy-Induced Sweet's Syndrome Treated with Infliximab
}

\author{
Brigita D. Smolovic ${ }^{a} \quad$ Mirjana D. Gajic-Veljic ${ }^{b} \quad$ Milos M. Nikolic ${ }^{b}$ \\ Damir F. Muhovic ${ }^{a}$ \\ ${ }^{a}$ Department of Gastroenterohepatology, Clinical Center of Montenegro, Faculty of Medicine, University of \\ Montenegro, Podgorica, Montenegro; ${ }^{b}$ Department of Dermatovenereology, Clinical Center of Serbia, Medical \\ School, University of Belgrade, Belgrade, Serbia
}

\section{Significance of the Study}

- Sweet's syndrome may be triggered by pregnancy. In cases of pregnant women with Sweet's syndrome and onset or relapse of Crohn's disease, the pregnancy might be a simultaneous trigger for both diseases. Sweet's syndrome can be treated with "off-label” use of infliximab.

\section{Keywords}

Sweet's syndrome · Pregnancy $\cdot$ Crohn's disease $\cdot$ Infliximab

\section{Abstract}

Objective: To present a case of relapsing and resistant Sweet's syndrome that developed during pregnancy together with an onset of Crohn's disease, showing complete resolution with the use of infliximab. Clinical Presentation and Intervention: A 30-year-old pregnant woman presented with fever, skin lesions, and diarrhea. Skin biopsy confirmed neutrophilic dermatosis and she was diagnosed with Crohn's disease after endoscopy. There was no recurrence of Sweet's syndrome outside of her pregnancy. During a previous pregnancy, while corticosteroids were ineffective, complete regression of skin lesions was achieved using infliximab. Conclusion: The "off-label" use of infliximab is beneficial for relapsing and resistant Sweet's syndrome.

(c) 2018 The Author(s)

Published by S. Karger AG, Basel

\section{Introduction}

Numerous etiological associations of Sweet's syndrome (SS) have been reported in the literature, but a concurrence of two conditions is infrequently reported. Immunological changes brought about by pregnancy can sensitize the immune system to elicit a secondary inflammatory reaction [1] leading to the onset or flare-up of Crohn's disease (CD) and/or SS. Recent data have also indicated that levels of proinflammatory cytokines become elevated in pregnancy, which might prove to be pivotal in the pathogenesis and severity of intestinal and extraintestinal symptoms [2]. There are not many recommendations on the use of TNFa inhibitors in the treatment of SS [3]. Here we present a patient with pregnancy-associated SS in an acute episode of CD, who achieved complete resolution of skin lesions after receiving infliximab in a relapsing and resistant form of SS. 


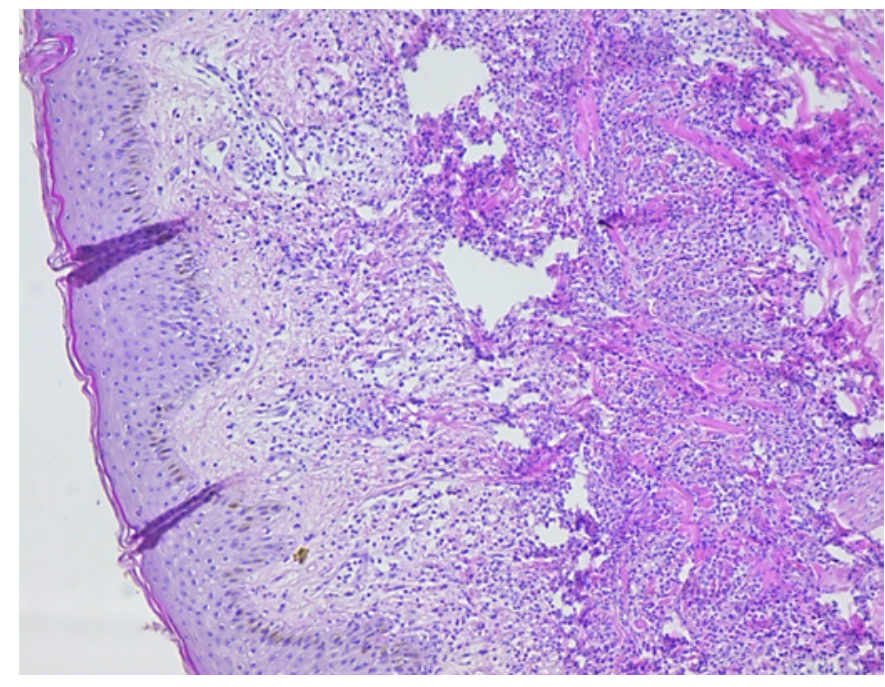

Fig. 1. Skin biopsy showed edema of the papillary dermis, a diffuse inflammatory infiltrate dominated by polymorphonuclear leukocytes, and necrosis of the reticular dermis (hematoxylin and eosin stain, $\times 100)$.
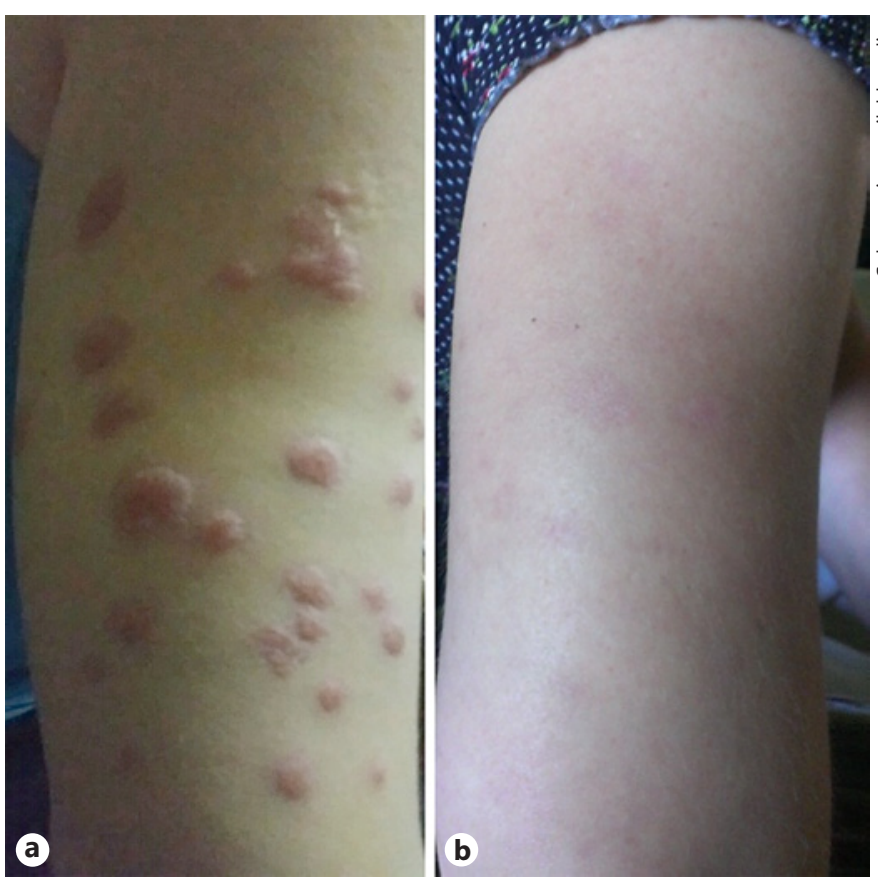

Fig. 2. Skin lesion. a Skin lesion before infliximab. b Disappearance of skin lesion after infliximab.

\section{Case Report}

A 30-year-old patient presented with fever and erythematous exudative plaques on both arms in the third month of her second pregnancy. Her past medical history did not reveal any previous disease. Over the period of several days, she had 4-5 mucous liquid stools. Her leukocyte count was $14.2 \times 10^{9} / \mathrm{L}$, with a neutrophilic predominance of $84.5 \%$. Her C-reactive protein was $114 \mathrm{mg} / \mathrm{L}$ and erythrocyte sedimentation rate was $94 \mathrm{~mm} / \mathrm{h}$. Skin biopsy showed an edema of the papillary dermis, a diffuse inflammatory infiltrate dominated by polymorphonuclear leukocytes, and a necrosis of the reticular dermis (Fig. 1). The initial diagnosis of the SS was made, and active CD was confirmed by sigmoidoscopy. After a consensus between specialists in gastroenterology, dermatology, and gynecology, corticosteroids (CS) were introduced, and both the cutaneous and intestinal manifestations disappeared. Over the next 9 years of follow-up, she became pregnant three more times, and skin lesions appeared in every instance. During the follow-up period, when she was not pregnant, she had two episodes of relapse of $\mathrm{CD}$, diagnosed with colonoscopy (A2L3B1 - by Montreal phenotype classification) and magnetic resonance enterography and treated with CS and azathioprine (which was discontinued shortly after because the patient developed an allergic reaction to it). Interestingly, both relapses were not followed by recurrence of SS. In the last pregnancy, her skin lesions (erythematous exudative plaques with a circular rim of vesicles on the upper extremities; Fig. 2a) did not respond to systemic CS. Remission of the SS and almost complete regression of the CD were achieved (Fig. 2b) after treatment with infliximab. At present, her CD is still in remission and no skin lesions have reoccurred.

\section{Discussion}

In the presence of skin lesions in pregnancy, SS should be kept in mind. SS may be associated with autoimmune and malignant diseases, as well as certain drugs. An extraordinarily high correlation between pregnancy and the onset of febrile neutrophilic dermatosis has been described in the literature [4]. In our case, both pregnancy and CD might be conditions that are linked to SS. Proinflammatory cytokines and acute phase proteins play an essential role in the pathogenesis of SS [1]. It is known that pregnancy can induce an upregulation of cytokines. The proper functioning of cytokine networks is essential for normal physiological homeostasis, and this role is emphasized by the fact that cytokine-mediated dysregulations are associated with several pregnancy complications which tend to exhibit changes in both local and systemic cytokine profiles [2]. In pregnancy, upregulated secretion of granulocyte colony-stimulating factor (G-CSF) and interleukin-6 (IL-6) secretion have been shown to play a crucial role in the induction of intestinal as well as dermal inflammation [5]. Therefore, pregnancy is considered as a possible trigger for SS as the skin lesions occurred only during the pregnancy. SS can recur in subsequent pregnancies [6], as it was the case here. The pregnancy can precede and/or can be associated with onset and/or flareup of CD [6]. It is known that the G-CSF which is upregulated in pregnancy can directly increase the number of 
neutrophils in the peripheral blood, which consecutively leads to inflammatory responses [1]. Moreover, significantly elevated inflammatory parameters appear to predict a potential risk of developing SS during an acute episode of IBD [1]. However, it is unlikely that the CD caused the SS in this case, as the patient did not have recurrence of SS concurrent with a new episode of CD when not pregnant. After the introduction of CS, cutaneous manifestations reliably disappeared in every pregnancy except in the last one. As the spontaneous healing of lesions may take weeks to months, topical or systemic treatment is recommended, especially in highly symptomatic cases [7]. Relapsing and resistant forms can be treated with immunomodulators or anti-TNF agents. Only limited use of TNFa inhibitors is recommended in the treatment of SS [3]. Infliximab is not approved for such indications at present and could be considered as off-label use [3]. Two studies found elevated levels of TNFa within the skin lesions present in SS $[8,9]$; thus, anti-TNF therapy might prove highly effective. We conclude that after the introduction of infliximab, a remission of both SS and CD was achieved, with an almost complete regression of skin lesions. In support of this finding, Rahier et al. [10] have also reported the excellent regression of cutaneous lesions after infliximab treatment in a patient with CD and SS. Further studies with infliximab are needed to define the role of this widely applicable and effective drug [3].

\section{Conclusion}

If two conditions associated with SS are present at the same time, it would be quite challenging to diagnose a trigger. In our case, it can be concluded that the pregnancy was the trigger for the SS and might be the cause of the onset of the CD. The "off-label" use of infliximab proved to be beneficial for the relapsing and resistant form of the SS.

\section{References}

1 Best J, Dechene A, Esser S, Gerken G, Canbay A. Pregnancy-associated Sweet's syndrome in an acute episode of ulcerative colitis. Z Gastroenterol. 2009 Aug;47(8):753-7.

2 Azizieh FY, Raghupathy RG. Tumor necrosis factor- $\alpha$ and pregnancy complications: a prospective study. Med Princ Pract. 2015;24(2): 165-70.

3 Rott S, Mrowietz U. The use of infliximab in dermatology. J Dtsch Dermatol Ges. 2007 Aug;5(8):655-60.

4 Cohen PR. Pregnancy-associated Sweet's syndrome: world literature review. Obstet Gynecol Surv. 1993 Aug;48(8):584-7.
5 Reuss-Borst MA, Pawelec G, Saal JG, Horny HP, Müller CA, Waller HD. Sweet's syndrome associated with myelodysplasia: possible role of cytokines in the pathogenesis of the disease. Br J Haematol. 1993 Jun;84(2): 356-8.

6 Yang CS, Teeple M, Muglia J, Robinson-Bostom L. Inflammatory and glandular skin disease in pregnancy. Clin Dermatol. 2016 MayJun;34(3):335-43.

7 Lima CD, Pinto RD, Góes HF, Salles SA, Vilar EA, Lima CD. Sweet's syndrome associated with Crohn's disease. An Bras Dermatol. 2017 Mar-Apr;92(2):263-5.
8 Cohen PR. Sweet's syndrome - a comprehensive review of an acute febrile neutrophilic dermatosis. Orphanet J Rare Dis. 2007 Jul; 2(1):34.

9 Patel R, Cafardi JM, Patel N, Sami N, Cafardi JA. Tumor necrosis factor biologics beyond psoriasis in dermatology. Expert Opin Biol Ther. 2011 Oct;11(10):1341-59.

10 Rahier JF, Lion L, Dewit O, Lambert M. Regression of Sweet's syndrome associated with Crohn's disease after anti-Tumour Necrosis Factor therapy. Acta Gastroenterol Belg. 2005 Jul-Sep;68(3):376-9. 